\title{
Klinefelter's Syndrome, Autoimmunity, and Associated Endocrinopathies
}

\author{
Key words: hypoandrogenism, hyperinsulinemia, immune system
}

\begin{abstract}
Klinefelter's syndrome (KS) is primary male hypogonadism caused by developmental testicular defects related to an underlying chromosomal abnormality. The usual karyotype in KS is 47, XXY (classical form), but 46, XY/47, XXY mosaicism is detected in approximately $10 \%$ of cases (1). The frequency of KS is about 1/500 males at conception, but half of the KS fetuses are lost during pregnancy, so the syndrome is present in $1 / 1,000$ live male newborns. The typical endocrine derangements observed in patients with KS include decreased secretion of androgens (testosterone, dihydrotestosterone, etc), increased plasma gonadotropins, and gynecomastia/male hypogonadism resulting from the relatively strengthened actions exerted by estrogens (estradiol, etc) compared with the actions exerted by androgens as known by increased estradiol/testosterone ratios. Another characteristic of KS is the concomitant occurrence of various autoimmune diseases such as rheumatoid arthritis, systemic lupus erythematosus (SLE), and thyroid autoimmune diseases. In this journal, Takeuchi et al (2) reported mixed connective tissue disease (MCTD), another associated autoimmune disease, in a case of KS.
\end{abstract}

\section{See also p 875.}

Although there have not yet been any adequate explanations for the concomitant occurrence of autoimmune diseases with KS, our understanding of this issue may be enhanced if we consider the following: 1 . The anti-autoimmune effects of androgens have been more definitively demonstrated in recent years both clinically and experimentally; 2 . It is well known that sexual dimorphism or feminine predominance is generally present in autoimmune diseases, including rheumatoid arthritis, SLE, primary biliary cirrhosis, Graves' disease and Hashimoto's thyroiditis (3); and 3. Sex hormones exercise their sex-specific functions based on the biological balance of counterpart effects between androgens and estrogens.

Acceptance of the theory that gonadal steroids regulate immune system function including the genesis of autoimmunity is growing $(3,4)$, and many investigators have reported animal experiments showing that administration of androgens suppresses the development of autoimmune diseases such as type 1 diabetes, Sjögren's syndrome, and $\operatorname{SLE}(3,5,6$,). Clinically, there is an interesting report describing a marked decrease in anti-DNA antibody titer following testosterone replacement therapy in a KS patient associated with SLE (7). In other studies, the thymus was proved to express androgen receptors, and thymus enlargement, occuring in animals that were castrated or in androgen withdrawal, was shown to have a central influence on the immune system $(3,4)$. Replacement with androgens in these animals causes thymocyte apoptosis and subsequent thymic atrophy. In addition to the central effects, androgens affect the production of cytokines such as transforming growth factor $\beta$ (TGF- $\beta$ ) and interferon $\gamma$ (IFN- $\gamma$ ). TGF- $\beta$, an important immunomodulator exerting antiproliferative effects on thymocyte and T cells, is upregulated at both mRNA and protein levels by androgens. IFN- $\gamma$, an accelerator of immune and autoimmune responses which is produced by the Th1 subset of helper $\mathrm{T}$ cells, is downregulated by androgens. By enhancing the expression of class I and class II MHC antigens in target cells, IFN- $\gamma$ facilitates autoimmune attack of the cells by effector lymphocytes. Also, as IFN- $\gamma$ promotes the differentiation of Th1 cells which is a producer of interleukin 2 (IL-2), the negative effects of androgens on IFN- $\gamma$ production could easily lead to reduced production of IL-2, the most potent amplifier in the immune cytokine network. These effects of androgens on cytokine production naturally lead to an attenuation of immune responses. Moreover, androgens also act on the $B$ cell system. While there is no direct action on peripheral $\mathrm{B}$ cells per se, androgens deplete pre-B cells in the bone marrow and diminish peripheral B cell numbers, ultimately suppressing autoantibody production (4).

As a matter of fact, Jefferies (8) has proposed that the combination of three factors (hypoandrogenism, mild adrenocortical insufficiency, and mycoplasma infection) is a possible cause of rheumatoid arthritis. If mild adrenocortical insufficiency is present, we cannot sufficiently respond to stress or sufficiently suppress early autoimmune reactions. It is a compelling idea to consider that autoimmune diseases may be chronically elicited by long-standing exposure of the host to antigens or infectious agents in the presence of an insidius adrenocortical insufficiency together with hypoandrogenism. On this point, the report from Takeuchi et al (2) is indeed excellent because it proved the presence of mild adrenocortical insufficiency in a patient based on reliable data that verified increased ACTH secretion, decreased circulatory dehydroepiandrosterone sulfate, and decreased urinary free cortisol. Thus, their report may serve as an important landmark in the understanding of the 
mechanisms of autoimmunity in KS.

More than three decades ago, diabetes mellitus (DM) was already reported as a disease frequently associated with KS (9-11), but the reports from that decade rarely distinguished DM types between type 1 (caused by autoimmunity) and type 2 (caused by insulin resistance). Now looking back at the past literature, the majority of KS patients reported to have DM seem to have had type $2 \mathrm{DM}$ presenting with hyperglycemia associated with secretion of considerable amounts of insulin (insulin resistance). Nonetheless, type $1 \mathrm{DM}$ can also occur in the presence of a sufficient causality related to autoimmunity based on hypoandrogenism, as mentioned above.

There are mutual relationships between androgen and insulin actions (12). In obesity and type 2 diabetes, hyperinsulinemia due to insulin resistance is associated with decreased testosterone levels, while hypogonadal males are frequently hyperinsulinemic or insulin resistant. For the latter, the treatment with testosterone induces reduction of insulin and leptin levels in parallel with a decrease of fat tissue mass. The mechanisms of both causal relationships (hyperinsulinemia inducing hypotestosteronemia and vice versa) are not clearly known and await further elucidation.

Norihiko AOKI, MD The Second Department of Internal Medicine, Kinki University School of Medicine, 377-2, Ohnohigashi, Osakasayama 589-8511

\section{References}

1) Grumbach MM, Conte FA. Classification of errors in sex differentiation. in: Williams Textbook of Endocrinology, Wilson JD, Foster DW, Kronenberg HM, Larsen PR, Eds. Saunders, Philadelphia, 9th edition, 1998: 1331-1337.

2) Takeuchi Y, Murata Y, Shintani J, et al. Klinefelter's syndrome accompanied by mixed connective tissue disease and diabetes mellitus. Intern Med 38: 875-881, 1999.

3) Olsen NJ, Kovacs WJ. Gonadal steroids and immunity. Endocrine Reviews 17: 369-384, 1996.

4) Olsen NJ. Androgens and T-cell development. Endo'99 Program \& Abstracts: San Diego, 1999: 43.

5) Sullivan DA, Rocha FJ, Sato EH. Influence of androgen therapy on lacrimal autoimmune disease in mouse model of Sjögren's syndrome. Advances in Mucosal Immunology 371: 1199-1202, 1995.

6) Toyoda H, Takei S, Formby B. Effect of 5- $\alpha$ dihydrotestosterone on Tcell proliferation of the female nonobese diabetic mouse. P.S.E.B.M. 213: 287-293, 1996.

7) Olsen NJ, Kovacs WJ. Case report: testosterone treatment of systemic lupus erythematosus in a patient with Klinefelter's syndrome. American Journal of Medical Sciences 310: 158-160, 1995.

8) Jefferies WM. The etiology of rheumatoid arthritis. Medical Hypotheses 51: 111-114, 1998.

9) Jackson IM, Buchanan KD, McKiddie MT, Prentice CR. Carbohydrate metabolism in Klinefelter's syndrome. J Endocrinol 35: 169-172, 1966.

10) Esmann V, Nielsen J, Petersen GB. A case of Klinefelter's syndrome with 48, XXXY and diabetes mellitus. Acta Med Scand 186: 27-33, 1969.

11) Hara $S$, Anderson RS, Bland W, Crump EP. Simultaneous occurrence of diabetes mellitus and Klinefelter's syndrome in two patients. J Natl Med Ass 62: 40-45, 1970.

12) Nieshlag E, Behre HM. Androgens, cardiovascular risk factors and atherosclerosis. in: Testosterone (editors: Nieschlag E, Behre HM, Eds Springer, New York, 2nd edition, 229-257, 1998). 